FSU-SCRI-97-122

November 1997

\title{
Accurate Scale Determinations for the Wilson Gauge Action
}

\author{
R.G. Edwards, U.M. Heller and T.R. Klassen \\ SCRI, Florida State University \\ Tallahassee, FL 32306-4130, USA
}

\begin{abstract}
Accurate determinations of the physical scale of a lattice action are required to check scaling and take the continuum limit. We present a high statistics study of the static potential for the $\mathrm{SU}(3)$ Wilson gauge action on coarse lattices $(5.54 \leq \beta \leq 6.0)$. Using an improved analysis procedure we determine the string tension and the Sommer scale $r_{0}$ (and related quantities) to $1 \%$ accuracy, including all systematic errors. Combining our results with earlier ones on finer lattices, we present parameterizations of these quantities that should be accurate to about $1 \%$ for $5.6 \leq \beta \leq 6.5$. We estimate the $\Lambda$-parameter of quenched QCD to be $\Lambda_{\overline{\mathrm{MS}}}=247(16) \mathrm{MeV}$.
\end{abstract}

\section{Introduction}

One of the technical but very important parts of a lattice simulation is the determination of the physical scale of the lattice. Only with accurate scale determinations can one check if the ratio of an observable to the physical scale has the theoretically expected scaling violations (e.g. order $a^{2}$ for the Wilson gauge action). This is required for the accurate and reliable continuum extrapolation of observables, and for their conversion into physical units to compare with (or predict) experiment.

Accurate scale determinations have been given new impetus by the recent progress in the Symanzik improvement program (see e.g. [1, 2]). In particular, the rather nontrivial, non-perturbative determination [3] (cf. also [4, 5]) of the $\mathrm{O}(a)$ coefficient in the Sheikholeslami-Wohlert (SW) quark action coupled to the Wilson gauge action has to be verified by accurate scaling checks. Only then would we feel confident to proceed with other steps in the improvement program.

It is known that the rho mass is a sensitive indicator of scaling violations, in particular of the $\mathrm{O}(a)$ violations of chiral symmetry that are generically present in a Wilson-type 
quark action like the SW action. The rho mass is an ideal quantity to use in scaling checks. Therefore we would rather not use it to set the scale, as is sometimes done. Instead we have to determine some other quantity sufficiently accurately to set the scale.

In pure gauge theory (or the quenched approximation of full QCD) the string tension $\sigma$ obtained from the static potential is such a quantity - in principle. In practice technical problems have often interfered with accurate determinations of this quantity. This is especially true on coarse lattices, $\beta<6.0$ for the Wilson gauge action. where, otherwise, scaling checks could be performed most accurately.

To our surprise we found that no accurate string tensions are known for the Wilson gauge action for $\beta<6.0$. The newest results we are aware of were presented in refs. [6, 7]. The errors are relatively large, and combined with string tensions on finer lattices the results do not really seem to lie on a smooth curve.

We have therefore decided to perform high statistics simulations of the static potential for the Wilson gauge action with $\beta \leq 6.0$, to determine the string tension and Sommertype scales [8] (the latter are also well-defined for full QCD). In sect. 2 we describe procedures to accurately calculate these quantities from the static potential. Sect. 3 gives the details of our simulations and the numerical results. In sect. 4 we present an accurate parameterization of our results for all $\beta$ in the range $5.6 \leq \beta \leq 6.5$. In sect. 5 we discuss the issue of scaling and extracting the $\Lambda$-parameter of QCD from the measured string tensions. We conclude in sect. 6 .

\section{Scale Determination with the Static Potential}

A standard method of fitting lattice Monte Carlo data for the static potential between an infinitely heavy quark and anti-quark is to use an ansatz of the form [9]

$$
V(\mathbf{r})=V_{0}+\sigma r-e\left[\frac{1}{\mathbf{r}}\right]+l\left(\left[\frac{1}{\mathbf{r}}\right]-\frac{1}{r}\right) .
$$

Here $\left[\frac{1}{\mathbf{r}}\right]$ denotes the tree-level lattice Coulomb term, viz,

$$
\left[\frac{1}{\mathbf{r}}\right]=4 \pi \int \frac{d^{3} \mathbf{k}}{(2 \pi)^{3}} \cos (\mathbf{k} \cdot \mathbf{r}) D_{00}(0, \mathbf{k})
$$

where $D_{00}(k)$ is the time-time component of the gluon propagator for the gauge action in question. Note that $l$ is an effective parameter introduced to model lattice artifacts beyond tree-level.

Clearly, eq. (2.1) is not a fundamental ansatz, since neither the running of the QCD coupling nor the lattice artifacts are taken into account properly (for a more ambitious attempt see [10]). This is a problem if one tries to extract $\Lambda_{\mathrm{QCD}}$ or other (intermediateto) short-distance aspects of the running coupling from potential data via an ansatz of the above or similar form.

\footnotetext{
${ }^{1}$ We use the standard notation $\beta \equiv 6 / g^{2}$ to parameterize the bare gauge coupling of the SU(3) Wilson plaquette action.
} 
We are here not interested in short-distance properties of the potential. Instead, we would like to extract a physical scale from the (intermediate- to) long-distance part of the potential.

Let us first consider determining the string tension $\sigma$, which, according to the flux tube picture and MC studies, is a well-defined quantity in pure gauge theory. When trying to determine only the string tension, the problems of the ansatz (2.1) at short distances become largely irrelevant. In practice we will always leave out the first few lattice points and check if the string tension stabilizes as we include in the fit only points with $r \geq r_{\min }$, with increasing $r_{\min }$.

According to the string picture the potential in pure gauge theory behaves for large distances as

$$
V(r)=V_{0}+\sigma r-\frac{e_{\mathrm{IR}}}{r}+\ldots
$$

If the transverse fluctuations of the QCD flux tube are described by the simplest bosonic string theory, then the "IR charge" takes on the universal value $e_{\mathrm{IR}}=\pi / 12$ in four spacetime dimensions [11]. Like others, we have observed that even with very good statistics (and smearing, etc) the intermediate- and long-distance part of lattice potential data can indeed always be described by an ansatz of the form (2.1) with $e=\pi / 12$.

To determine the string tensions we therefore perform three types of fits:

- 4-parameter fits to (2.1).

- 3-parameter fits to (2.1) with $e=\pi / 12$ fixed.

- 2-parameter fits to (2.1) with $e=\pi / 12$ and $l=0$ fixed.

As $r_{\text {min }}$ increases for the various fits, they should reach consistent values for the string tension. This asymptotic value is expected to emerge first for the 4-parameter fit, next for the 3- and finally for the 2-parameter fit. The demand that all three fits give a consistent value should allow an accurate and reliable determination of the string tension. We should remark that similar ideas were used in the string tension determinations by the Bielefeld group [12].

Although the method just sketched works quite well, as we will see, there are clearly drawbacks to using the string tension to set the scale. First of all, of course, in full QCD the string tension is not well-defined, due to string breaking. Secondly, due to its long-distance nature, one needs the potential in a region where errors are becoming large.

To avoid these problems Sommer [8] suggested to define an intermediate distance scale $r_{c}$ via the force between a heavy quark and anti-quark,

$$
r_{c}^{2} V^{\prime}\left(r_{c}\right)=r_{c}^{2} F\left(r_{c}\right) \stackrel{!}{=} c
$$

for some suitably chosen real number $c$. The question is how to implement this idea in practice to extract $r_{c}$ from lattice data. 
One approach is to define the force and its error via discrete (correlated) differences of pairs of points,

$$
F\left(r_{I}\right) \equiv \frac{\left|V\left(\mathbf{r}_{1}\right)-V\left(\mathbf{r}_{2}\right)\right|}{\left|\mathbf{r}_{1}-\mathbf{r}_{2}\right|},
$$

where $r_{I}$ is chosen [B] to eliminate tree-level lattice artifacts,

$$
\frac{1}{r_{I}^{2}} \equiv-\frac{\left|\left[\frac{1}{\mathbf{r}_{1}}\right]-\left[\frac{1}{\mathbf{r}_{2}}\right]\right|}{\left|\mathbf{r}_{1}-\mathbf{r}_{2}\right|}
$$

In practice one finds that the force values from different pairs of points around $r_{c}$ still do not fall on a smooth curve, due to remaining lattice artifacts. When interpolating the force to obtain $r_{c}$ one therefore ends up with relatively large errors.

One can do better (see e.g. 113]) by defining $r_{I}$ via the corrected (more continuum-like) potential data,

$$
V_{c}(\mathbf{r}) \equiv V_{\mathrm{MC}}(\mathbf{r})+(e-l)\left(\left[\frac{1}{\mathbf{r}}\right]-\frac{1}{r}\right)
$$

as

$$
\frac{\partial V_{c}}{\partial r}\left(r_{I}\right)=\sigma+\frac{e}{r_{I}^{2}} \stackrel{!}{=} \frac{\left|V_{c}\left(\mathbf{r}_{1}\right)-V_{c}\left(\mathbf{r}_{2}\right)\right|}{\left|\mathbf{r}_{1}-\mathbf{r}_{2}\right|},
$$

where $\sigma, e$ and $l$ are taken from a fit to the ansatz (2.1). By (slight) abuse of notation we are also using $V_{c}(r) \equiv V_{0}+\sigma r-e / r$ on the left side of eq. (2.8) to denote the continuum part of (2.1).

Taking the last idea to its logical conclusion we will use the following method to determine $r_{c}$ : Perform a correlated fit of the potential data to an ansatz of the form (2.1) — but only locally, using data between some $r_{\min }$ and $r_{\max }$ close to $r_{c}$. We then obtain $r_{c}$ from the continuum part of the ansatz as

$$
r_{c}=\sqrt{\frac{c-e}{\sigma}}
$$

Since we are not performing a global fit of the potential data the precise from of the (continuum part of the) ansatz should not be crucial. W We can estimate the systematic error of $r_{c}$ by varying $r_{\min }$ and $r_{\max }$, and by fixing $e=\pi / 12$. Similarly, we can get an idea of the effect of lattice artifacts beyond tree-level by comparing free $l$ with $l=0$ fits.

The nice feature of this strategy is that we can avoid taking the numerical derivatives required for the force, while still using only local information about the potential and being able to take lattice artifacts beyond tree-level into account. The fits required are of the same kind that one performs to determine the string tension; one just has to consider smaller intervals $\left[r_{\min }, r_{\max }\right]$ around $r_{c}$.

\footnotetext{
${ }^{2}$ One would therefore expect the general 4-parameter fit to be somewhat over-parameterized. Indeed, we find that for $r_{c}$ fits $e$ and $\sigma$ are strongly (anti-) correlated.
} 


\begin{tabular}{|l|c|c|c|}
\hline$\beta$ & Volume & $L(\mathrm{fm})$ & $N_{\text {confgs }}$ \\
\hline 6.0 & $16^{3} \cdot 32$ & 1.5 & 4000 \\
5.85 & $16^{3} \cdot 32$ & 2.0 & 8000 \\
5.7 & $16^{3} \cdot 32$ & 2.6 & 4000 \\
5.6 & $12^{4}$ & 2.6 & 2000 \\
& $8^{4}$ & 1.7 & 4630 \\
5.54 & $12^{4}$ & 2.9 & 2000 \\
\hline
\end{tabular}

Table 1: Volume, spatial extent and number of configurations used in our simulations.

\section{Simulations and Results}

We performed simulations at five couplings $\beta \leq 6.0$ for the $\mathrm{SU}(3)$ Wilson plaquette action. Our updating algorithm alternates microcanonial over-relaxation and heatbath steps acting on $\mathrm{SU}(2)$ subgroups [14] (typically in a 3:1 ratio per sweep). In all cases we generated several thousand (essentially) independent configurations on lattices whose spatial extent is at least $1.5 \mathrm{fm}$; cf. table 1. We should remark that a subset of the $32 \cdot 16^{3}$ configurations at $\beta=5.7$ and 5.85 are also being used for spectrum calculations with the non-perturbatively improved SW quark action [5].

The static potential is obtained in the standard manner from (smeared) Wilson loops $W(\mathbf{r}, t)$ via the effective local masses

$$
V_{t}(\mathbf{r}) \equiv \ln \frac{W(\mathbf{r}, t)}{W(\mathbf{r}, t+1)}
$$

To reach plateaus for small $t$ we perform APE smearing [15] on the spatial link variables. This amounts to adding the spatial staples to a suitable multiple of the given link,

$$
U_{k}(x) \rightarrow \alpha U_{k}(x)+\sum_{l: l \neq k} U_{l}(x) U_{k}(x+\hat{l}) U_{-l}(x+\hat{l}+\hat{k}),
$$

and projecting back onto $\mathrm{SU}(3)$. This procedure is then iterated $n$ times.

Note that (modulo details of the $\mathrm{SU}(3)$ projection) the above procedure is essentially equivalent to a more intuitive "covariant gaussian smearing" in terms of the transverse laplacian $\Delta_{\perp}$

$$
U_{k}(x) \rightarrow\left(1+\epsilon \Delta_{\perp}\right)^{n} U_{k}(x) \approx \mathrm{e}^{\epsilon n \Delta_{\perp}} U_{k}(x) .
$$

In momentum space the exponential becomes a gaussian, at least in the free case. The relation between $\epsilon$ and $\alpha$ is $\epsilon \approx 1 /(4+\alpha)$.

This way of writing the smearing is useful, because it shows that as long as $\epsilon$ is small the actual amount of smearing will only depend on the product $\epsilon n$. Dimensional

\footnotetext{
${ }^{3}$ We do not write an exact equality here, because of possibly different implementations of the projection, and the question of whether one uses the initial or the "updated" gauge field in the covariant laplacian.
} 
analysis then also shows that the same amount of smearing, in physical units, corresponds to scaling $\epsilon n \propto 1 / a^{2}$ as one varies the lattice spacing. One therefore has to optimize the smearing only at one coupling and can then use this scaling to find a (near-) optimal smearing at other lattice spacings. We find that $\epsilon n \approx 4$ is a good smearing for $\beta=6.0$. Even for large $r$ ground state overlaps are then at least $85 \%$.

It is important to have off-axis $\mathbf{r}$ to accurately determine the string tension and $r_{c}$. We measured the potential at multiples of the lattice vectors $(1,0,0),(1,1,0),(1,1,1)$ and $(2,1,0)$.

Our fitting procedure was sketched in sect. 2. For a given fit, i.e. for a given ansatz, $t$ in eq. (3.1), as well as $r_{\min }$ and $r_{\max }$, we use a single elimination jackknife procedure (after suitable binning of the data) to obtain the errors of all quantities of interest. The final value and error we quote is obtained as follows. For each fit ansatz (with 2, 3, or 4 parameters, cf. sect. 2) and $t$ we consider the best few fits (five or less, say), that satisfy a minimal "goodness" criterion. Our "goodness" function is typically the product of the confidence level and the number of degrees of freedom of the fit. We then combine all the "good" fits with a weight that depends on the goodness and the error of the quantity in question.

This procedure is semi-automated to eliminate subjective bias. It is hard to completely automate this process. For example, it can happen (e.g. if the smearing is not optimal) that fits from neighboring pairs of time slices seem roughly equally "good", but differ significantly in their estimate of the quantity under consideration. In such cases it seems preferable to invoke human intelligence and experience to decide what weight to assign to the different fits.

Overall our analysis procedure is conservative, because we do not simply take the one "best" fit according to some necessarily somewhat subjective criterion. By always taking several fits into account we find that our final numbers and error bars are quite stable under changes of the goodness function or of the cuts on the minimal required goodness of a fit. Our final error is never smaller than that of any single good fit. In case one fit is much "better" than the others, our error and central value will be close to the values of this fit; in general our error bar is significantly larger, and our central value somewhere between that of the different fits.

Using this procedure we have measured the string tension and several $r_{c}$. The "standard" choice of $r_{c}$ [8] corresponds to $c=1.65$. Slightly inconsistently, but in accord with accepted conventions, we will denote this choice by $r_{0}$. It corresponds to $r_{0} \approx 0.50 \mathrm{fm}$ (perhaps a bit less); which is around the region where phenomenological potentials are best determined. On fine lattices it also corresponds to the region where the potential can be very accurately measured. On lattices coarser than $0.1 \mathrm{fm}$ this choice becomes increasingly impractical, however, because $r_{0}$ is then quite small in lattice units. We have therefore decided to measure $r_{4}$ and $r_{6}$ in addition to $r_{0}$. We note that $r_{6} \approx 1.0 \mathrm{fm}$.

\footnotetext{
${ }^{4}$ The situation is more complicated for improved gauge actions, due to the contributions from "ghost states" to correlation functions at small times.

${ }^{5}$ For the simulations on $8^{4}$ lattices at $\beta=5.6$ we measured several more $\mathbf{r}$ values. This case is also exceptional in that we used an updating algorithm that alternates over-relaxation and Metropolis steps.
} 

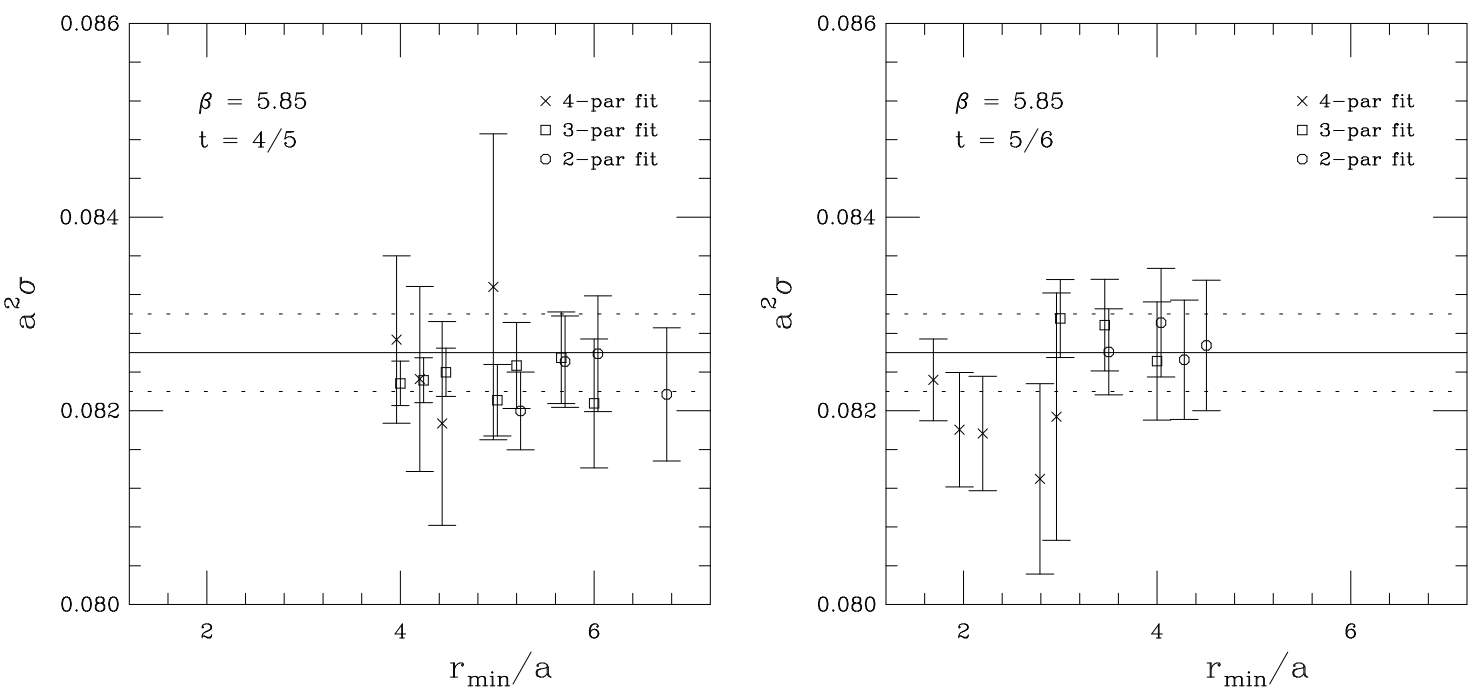

Figure 1: String tension for $\beta=5.85$ as a function of $r_{\min }$ for different "good" fits and using time slices $t=4 / 5$ and $t=5 / 6$. The solid and dashed lines denote our final value and its error, respectively.

\begin{tabular}{|l|l|l|c|c|}
\hline$\beta$ & \multicolumn{1}{|c|}{$a \sqrt{\sigma}$} & \multicolumn{1}{|c|}{$r_{0} / a$} & $r_{4} / a$ & $r_{6} / a$ \\
\hline 5.54 & $0.5727(52)$ & $2.054(13)$ & $3.381(21)$ & $4.187(27)$ \\
5.6 & $0.5064(28)$ & $2.344(8)$ & $3.814(15)$ & $4.718(23)$ \\
5.7 & $0.3879(39)$ & $2.990(24)$ & $4.926(35)$ & $6.128(42)$ \\
5.85 & $0.2874(7)$ & $4.103(12)$ & $6.733(17)$ & $8.340(24)$ \\
6.0 & $0.2189(9)$ & $5.369(9)$ & $8.831(21)$ & $10.89(3)$ \\
\hline
\end{tabular}

Table 2: Our results for the string tension and Sommer-type scales.

A final remark about the $r_{c}$ determinations. Recall that in this case we vary $r_{\min }$ and $r_{\max }$ around $r_{c}$. We find, perhaps not too surprisingly, that the dependence on $r_{\max }$ is always small, in most cases negligible.

Some of our results are illustrated in figures 1 - 3. Note in particular the stability of our determination of $r_{0}$ in figure 3, even for values of $r_{\min }$ larger than $r_{0}$ itself! Such fits are not included in our final analysis, but we regard this as a sign of the stable and conservative nature of our analysis strategy. Our final results are collected in table 2. The relatively large errors in the $\beta=5.7$ case are presumably due to non-optimal smearing; we did not follow the guideline described earlier (yet).

As explained above, we believe that the values quoted include all systematic errors - except possibly for one source we have not discussed so far: finite volume effects. Previous studies for $\beta \geq 6.0$ indicate that a spatial extent of about $1.5 \mathrm{fm}$ is sufficient to suppress finite volume errors in the string tension to the level of about one percent or 


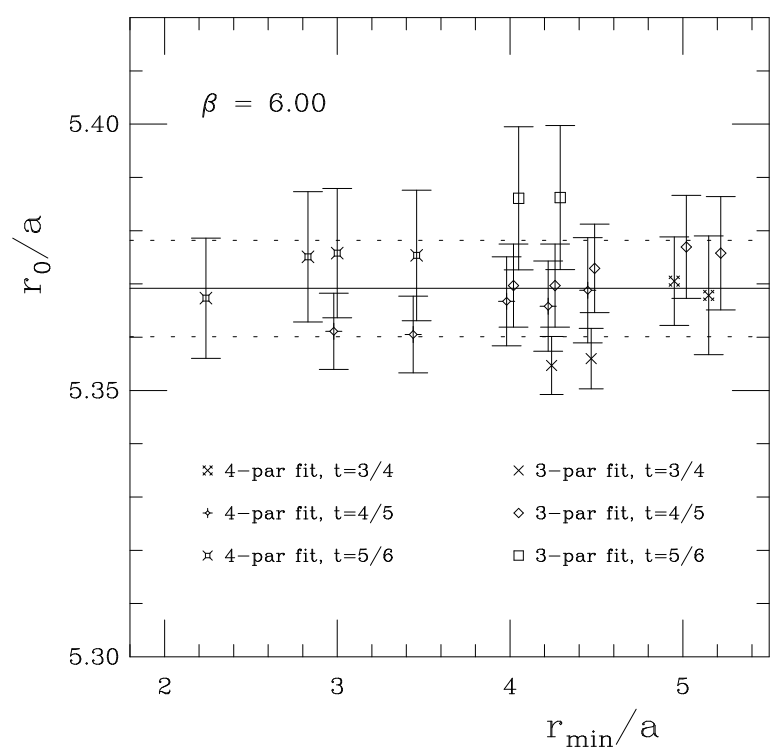

Figure 2: Sommer scale $r_{0}$ for $\beta=6.0$ as a function of $r_{\min }$ for various "good" fits. The solid and dashed lines denote our final value and its error, respectively.

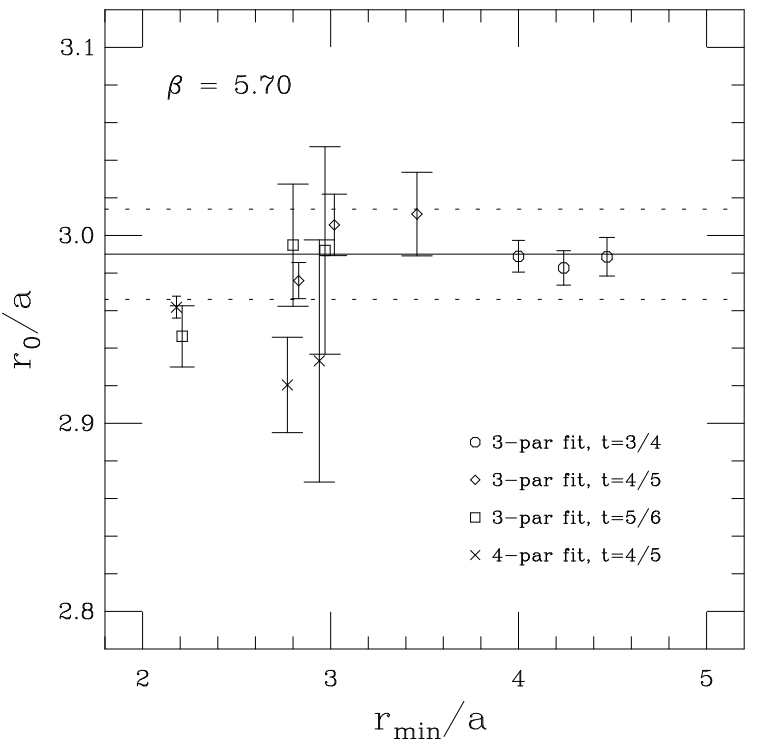

Figure 3: Sommer scale $r_{0}$ for $\beta=5.70$ as a function of $r_{\min }$ for various "good" fits. The solid and dashed lines denote our final value and its error, respectively. Note that the 3 -parameter fits with $t=3 / 4$ have $r_{\min }>r_{0}$. These fits were not included in our final error estimate; they are shown only for illustration. 


\begin{tabular}{|l|l|l|l|}
\hline Scale & $8^{4}(1.7 \mathrm{fm})$ & $12^{4}(2.6 \mathrm{fm})$ & Change \\
\hline$a \sqrt{\sigma}$ & $0.5004(23)$ & $0.5064(28)$ & $+1.2 \% \pm 0.7 \%$ \\
$r_{0} / a$ & $2.355(7)$ & $2.344(8)$ & $-0.5 \% \pm 0.5 \%$ \\
$r_{4} / a$ & $3.867(10)$ & $3.814(15)$ & $-1.4 \% \pm 0.5 \%$ \\
$r_{6} / a$ & $4.787(14)$ & $4.718(23)$ & $-1.5 \% \pm 0.6 \%$ \\
\hline
\end{tabular}

Table 3: Check of finite volume effects for $\beta=5.6$.

less [16]. To explicitly check this for our determinations, we have repeated the simulations at $\beta=5.6$ on $8^{4}$ in addition to $12^{4}$ lattices. The comparison is shown in table 3 . It would seem that within our errors there are significant finite volume effects on the $1-1.5 \%$ level, except for $r_{0}$, which seems to be much less affected (not too surprisingly). The sign of the change of the string tension is as expected (from earlier studies) for finite volume effects. That the $r_{c}$ move in the opposite direction is also expected.

In any case, for coarse lattices there is no problem in using a sufficiently large volume to suppress finite volume effects to significantly less than one percent. This is true for the values in table 2, except perhaps for $\beta=6$.0. We will return to this point in the next section.

\section{Parameterizing the Scale}

The string tension is one of the quantities that has traditionally been used to check perturbative scaling. It is known that, on a quantitative level, the string tension follows neither two- or three-loop scaling, at least for the lattice spacings we are interested in (we will see this explicitly below). Allton [17] suggested that this is due to $a^{2}, g^{2} a^{2}, \ldots, a^{4}, \ldots$ lattice artifacts, that are unavoidable if one determines the string tension with the Wilson gauge action.

He therefore proposed [17] to fit the string tension to an ansatz of the form

$$
(a \sqrt{\sigma})(g)=f\left(g^{2}\right)\left(1+c_{2} \hat{a}(g)^{2}+c_{4} \hat{a}(g)^{4}+\ldots\right) / \frac{\Lambda}{\sqrt{\sigma}}, \quad \hat{a}(g) \equiv \frac{f\left(g^{2}\right)}{f\left(g^{2}(\beta=6)\right)},
$$

in terms of the fit parameters $\Lambda / \sqrt{\sigma}, c_{2}, c_{4}, \ldots$. Note that $\hat{a}(g)$ has been normalized to 1 for $\beta=6.0$; we will also use this convention in other coupling schemes considered later. In the above $f\left(g^{2}\right)$ is the universal two-loop scaling function of $\mathrm{SU}(3)$ gauge theory,

$$
f\left(g^{2}\right) \equiv\left(b_{0} g^{2}\right)^{-\frac{b_{1}}{2 b_{0}^{2}}} \exp \left(-\frac{1}{2 b_{0} g^{2}}\right), \quad b_{0}=\frac{11}{(4 \pi)^{2}}, \quad b_{1}=\frac{102}{(4 \pi)^{4}} .
$$

Recall that if scaling holds with a scaling function $f$ in a scheme $S$, then $\Lambda_{S}=q f\left(g_{S}^{2}(q)\right)$ is a constant, the $\Lambda$-parameter of the scheme.

On theoretical grounds one might want to add $g^{2} \hat{a}(g)^{2}, \ldots$ corrections to the terms shown in eq. (4.1), but in practice this does not turn out to be useful (however, we 


\begin{tabular}{|l|l|l|l|l|}
\hline$\beta$ & \multicolumn{1}{|c|}{$a \sqrt{\sigma}$} & $r_{0} / a$ & Volume & Reference \\
\hline 5.54 & $0.5727(52)$ & $2.054(13)$ & $12^{4}$ & This work \\
5.6 & $0.5064(28)$ & $2.344(8)$ & $12^{4}$ & This work \\
5.7 & $0.3879(39)$ & $2.990(24)$ & $16^{3} \cdot 32$ & This work \\
5.85 & $0.2874(7)$ & $4.103(12)$ & $16^{3} \cdot 32$ & This work \\
6.0 & $0.2189(9)$ & $5.369(9)$ & $16^{3} \cdot 32$ & This work \\
& $0.2182(16)$ & & $16^{4}$ & Perantonis/Michael [19] \\
& $0.2184(19)$ & $5.35(+2)(-3)$ & $16^{4}$ & SESAM [13] \\
& $0.2209(23)$ & & $32^{4}$ & Bali/Schilling/Hoeber [20] \\
& $0.2154(50)$ & $5.47(11)$ & $16^{3} \cdot 48$ & UKQCD [21] \\
6.2 & $0.1610(9)$ & & $32^{4}$ & Bali/Schilling/Hoeber [20] \\
& $0.1604(11)$ & $7.37(3)$ & $32^{4}$ & SESAM [13] \\
& $0.1608(23)$ & $7.29(17)$ & $24^{3} \cdot 48$ & UKQCD [21] \\
6.4 & $0.1214(12)$ & $9.89(16)$ & $32^{4}$ & Bali/Schilling [16, 18] \\
& $0.1218(28)$ & $9.75(17)$ & $32^{3} \cdot 64$ & UKQCD [21] \\
6.5 & $0.1068(9)$ & $11.23(21)$ & $36^{4}$ & UKQCD [22, 23] \\
\hline
\end{tabular}

Table 4: Selected world results for the string tension and $r_{0}$.

have checked that the $\Lambda$-parameter obtained from fits is stable when using such terms in (4.1)).

In table 4 we have collected some results for the string tension and $r_{0}$. We are not showing data for $\beta=6.6$ and 6.8 , since they might be affected by finite volume errors [18]. Looking at the different volumes employed for the data shown in table 1 , it seems quite clear that finite volume errors in the string tension are on the one percent or less level for all $\beta$ (cf. also our remarks in sect. 3 and the discussion in the references quoted in the table).

These data are well fitted by an ansatz of the form (4.1) using the two-loop scaling function for the bare coupling. [. We obtain for the 12 degree of freedom fit of the string tension with all data in the table,

$$
\begin{aligned}
\frac{\Lambda}{\sqrt{\sigma}} & =0.01338(12), \\
c_{2} & =0.195(16) \\
c_{4} & =0.0562(45)
\end{aligned}
$$

with a confidence level of $Q=0.19$. Adding an $\hat{a}(g)^{6}$ term in the ansatz gives a $Q$ of 0.24 . For the three-parameter fit of $r_{0}$ we obtain $Q=0.12$.

When leaving out the $\beta=5.54$ point from the fits, the confidence levels jump up to $Q=0.9$ for three-parameter fits of both $\sqrt{\sigma}$ and $r_{0}$. This might indicate that the

\footnotetext{
${ }^{6}$ In the next section we will discuss fits in terms of other couplings, that we consider to be more physical. Here our aim is the purely pragmatic one of providing an explicit, analytic parameterization of the data, for which, it turns out, the bare coupling is just as suited as any other one.
} 
determination of these quantities at $\beta=5.54$ is increasingly tainted by lattice artifacts that are not modeled with sufficient accuracy by our ansatz. We find some signs of this in our potential fits.

We would like to present a parameterization of the data that reproduces the central values within one percent and one standard deviation, whichever is smaller (considering the tiny error bars in $\sqrt{\sigma}$ at $\beta=5.85$ we allow a difference of, say, 1.5 standard deviations there). This is difficult to achieve with the $\beta=5.54$ point, so we will do so only for the interval $5.6 \leq \beta \leq 6.5$. The following parameterizations satisfy these constraints:

$$
\begin{aligned}
& (a \sqrt{\sigma})(g)=f\left(g^{2}\right)\left(1+0.2731 \hat{a}(g)^{2}-0.01545 \hat{a}(g)^{4}+0.01975 \hat{a}(g)^{6}\right) / 0.01364 \\
& \left(a / r_{0}\right)(g)=f\left(g^{2}\right)\left(1+0.2106 \hat{a}(g)^{2}+0.05492 \hat{a}(g)^{4}\right) / 0.01596
\end{aligned}
$$

The representation of the string tension is shown in figure 4 , together with the data from table 4 and results obtained earlier for $\beta<6.0$.

\section{$5 \quad$ Scaling and $\Lambda_{\overline{\mathrm{MS}}}$}

One can consider taking the above much more seriously than just as a way of parameterizing the data. That is, one can try to extract the $\Lambda$-parameter of QCD from it. The statistical uncertainties of the fit parameters are very small, about $1 \%$ for $\Lambda / \sqrt{\sigma}$ and slightly larger for $\Lambda r_{0}$. However, before taking this seriously as a determination of the $\Lambda$ parameter, we have to consider systematic errors.

The most important question is whether the $\Lambda$-parameter extracted from a two-loop fit with the bare coupling is stable when going to three-loops, and when considering other schemes for the coupling. It is believed that perturbative scaling holds better when using an "improved" coupling [24]. The precision data for the string tension that are now available for a wide range of lattice spacings provide an excellent testing ground of these ideas.

We will consider the $E$-scheme [25], the $V$-scheme [26, 24], and the $\overline{\mathrm{MS}}$ scheme. In the $E$-scheme ( $E$ for energy) one uses the leading perturbative expansion of the expectation value of the plaquette, $W_{11}$, to define $g_{E}^{2}$ for $\mathrm{SU}(N)$ gauge theory via

$$
1-W_{11}=w_{1} g^{2}+w_{2} g^{4}+\ldots \equiv w_{1} g_{E}^{2}, \quad w_{1}=\frac{N^{2}-1}{8 N} .
$$

In practice one uses the value of the plaquette measured in a Monte Carlo simulation on the left hand side.

To obtain the coupling in the $V$-scheme, originally defined via the static potential, we use the expansion of the logarithm of the plaquette to second order to first define the "plaquette coupling" $g_{P}^{2}(q)$ [24]. Using the measured value of the plaquette, $g_{P}^{2}(q)$ is obtained by solving, for pure $\mathrm{SU}(N)$,

$$
-\ln W_{11}=w_{1} g_{P}^{2}(q)\left[1-2 b_{0} \ln \left(\frac{6.711706}{a q}\right) g_{P}^{2}(q)\right] .
$$




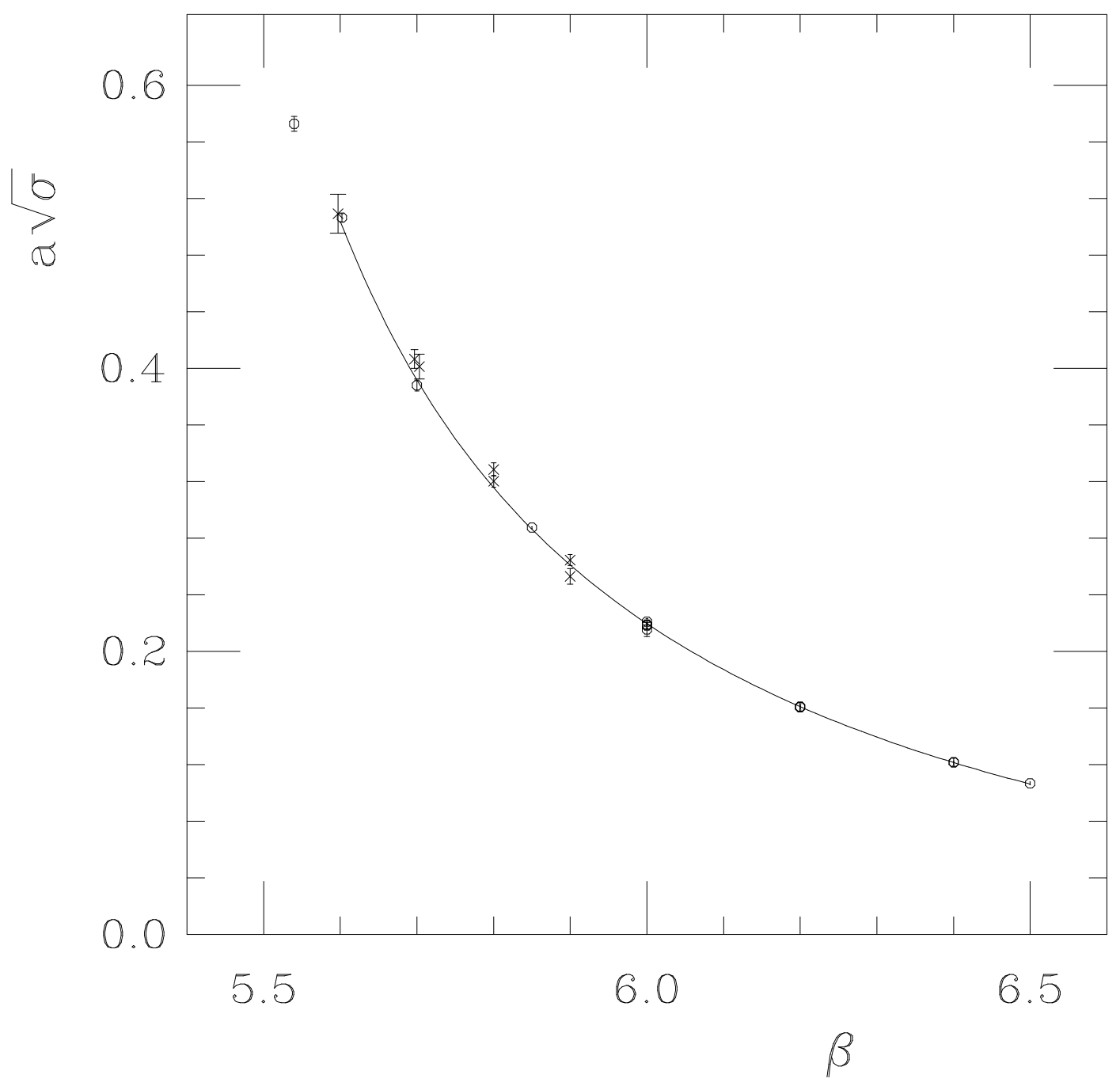

Figure 4: Plot of the parameterization (4.4) of the string tension as a function of $\beta=6 / g^{2}$. Also shown are the data from table 1 (o) that lead to the parameterization, as well as earlier results $(x)$ for coarse lattices from refs. [6, 7]. For clarity a few data points have been slightly displaced around their actual $\beta$ value. 


\begin{tabular}{|c|r|c|}
\hline Scheme & \multicolumn{1}{|c|}{$c_{S}$} & $\Lambda_{\overline{\mathrm{MS}}} / \Lambda_{S}$ \\
\hline bare & 0.189604 & 28.80934 \\
$E$ & 0.011629 & 13.88031 \\
$V$ & -0.136923 & 0.625192 \\
$\overline{\mathrm{MS}}$ & -0.012631 & 1 \\
\hline
\end{tabular}

Table 5: Three-loop coefficient $c_{S}=\left(b_{1}^{2}-b_{0} b_{2, S}\right) /\left(2 b_{0}^{3}\right)$ of the scaling function and $\Lambda$ parameter for pure $\mathrm{SU}(3)$ gauge theory in various schemes.

The optimal scale $q$ to extract the coupling, according to [24], is given by the value $q^{\star}=3.4018 / a$ [24, 10].

By construction $g_{P}^{2}=g_{V}^{2}+\mathrm{O}\left(g_{V}^{6}\right)$. The exact coefficient of the third order term is now also known (cf. [27, 28, 29] with the last missing ingredient recently provided in [30]), so that we can extract $g_{V}^{2}$ to third order from $g_{P}^{2}$ defined above:]

$$
g_{V}^{2}\left(q^{\star}\right)=g_{P}^{2}\left(q^{\star}\right)\left[1+\frac{2.814089}{(4 \pi)^{2}} g_{P}^{2}\left(q^{\star}\right)^{4}\right],
$$

for pure $\mathrm{SU}(3)$ and with $q^{\star}$ as above. We can also obtain $g_{\overline{\mathrm{MS}}}^{2}$ to third order from $g_{P}^{2}$, for which we will use the BLM [32] improved relation

$$
g_{\overline{\mathrm{MS}}}^{2}\left(\mathrm{e}^{-5 / 6} q^{\star}\right)=g_{P}^{2}\left(q^{\star}\right)\left[1+\frac{1}{2 \pi^{2}} g_{P}^{2}\left(q^{\star}\right)^{2}+\frac{0.9546933}{(4 \pi)^{2}} g_{P}^{2}\left(q^{\star}\right)^{4}\right] .
$$

In all four schemes the three-loop running of the coupling is known (see [28, 29] for the bare, e.g. [29] for the $E$-, [30] for the $V$-, and [31] for the $\overline{\mathrm{MS}}$-scheme). The scheme-dependent three-loop correction to the two-loop scaling function amounts to a multiplicative factor of the form $1+c_{S} g_{S}^{2}$. The value of the coefficient $c_{S}$ in various schemes is given in table 5, together with the $\Lambda$-parameters. Our ansatz for a three-loop fit of the string tension is therefore of the form

$$
(a \sqrt{\sigma})\left(g_{S}(q)\right)=\left(1+c_{S} g_{S}^{2}(q)\right) f\left(g_{S}^{2}(q)\right)\left[1+c_{2} \hat{a}\left(g_{S}(q)\right)^{2}+c_{4} \hat{a}\left(g_{S}(q)\right)^{4}\right] / \frac{\Lambda_{S}}{a q \sqrt{\sigma}} .
$$

Ultimately, once better data for $r_{0}$ are available on fine lattices, these fits should also be performed for $r_{0}$. At present, however, the data for the string tension are significantly better, overall.

For each scheme we have performed fits using a two-loop and a three-loop ansatz. For the $V$ and $\overline{\mathrm{MS}}$ cases the coupling is then also extracted consistently to second, respectively, third order from the measured value of the plaquette. We find that all data

\footnotetext{
${ }^{7}$ Since the third order expansion of the plaquette is now also known in terms of $g_{V}^{2}$, one could try to obtain the latter directly from the plaquette, without the detour via $g_{P}^{2}$. However, it turns out that the cubic equation for $g_{V}^{2}$ has no physical, i.e. real and positive, solutions for $\beta \leq 5.7$. The detour via $g_{P}^{2}$ is therefore to be preferred.
} 


\begin{tabular}{|c|c|c|c|c|c|c|}
\hline Scheme & Order & $g_{S}^{2}(q)$ & $a q$ & $c_{2}$ & $\Lambda_{S} /(a q \sqrt{\sigma})$ & $\Lambda_{\overline{\mathrm{MS}}}(\mathrm{MeV})$ \\
\hline bare & 2 & $6 / \beta$ & 1 & $0.171(17)$ & $0.01325(12)$ & $178(2)$ \\
& 3 & $6 / \beta$ & 1 & $0.157(16)$ & $0.01550(14)$ & $208(2)$ \\
\hline$E$ & 2 & $3\left(1-W_{11}\right)$ & 1 & $0.080(6)$ & $0.03817(21)$ & $246(1)$ \\
& 3 & $3\left(1-W_{11}\right)$ & 1 & $0.078(6)$ & $0.03864(21)$ & $249(1)$ \\
\hline$V$ & 2 & $g_{P}^{2}(q)$ & 3.4018 & $0.057(6)$ & $0.2604(13)$ & $258(1)$ \\
& 3 & eq. (5.3) & 3.4018 & $0.130(8)$ & $0.2449(15)$ & $242(1)$ \\
\hline$\overline{\mathrm{MS}}$ & 2 & $g_{P}^{2}\left(q^{\star}\right)+\frac{1}{2 \pi^{2}} g_{P}^{4}\left(q^{\star}\right)$ & 1.4784 & $0.074(6)$ & $0.3539(19)$ & $243(1)$ \\
& 3 & eq. (5.4) & 1.4784 & $0.072(6)$ & $0.3647(19)$ & $251(1)$ \\
\hline
\end{tabular}

Table 6: Fit results for the string tension data $\beta \geq 5.6$ in various schemes to an ansatz of the form (5.5) (the values of the fit parameter $c_{4}$ are not shown). The order indicates which scaling function was used (two- or three-loop) and, for the $V$ - and $\overline{\mathrm{MS}}$-schemes, to which order the coupling was extracted from the measured plaquette. We assume $\sqrt{\sigma}=465 \mathrm{MeV}$ in converting to physical units. Details are in the text.

can be fitted reasonably well in all schemes with either the two- or the three-loop ansatz. In either order the fits in the different schemes are of comparable quality.

When leaving out small $\beta$ points the fit parameters are essentially stable within errors in all cases. This is particularly impressive for $\Lambda_{S} /(a q \sqrt{\sigma})$, which is stable on the $1 \%$ level even when all $\beta<6.0$ points are left out of the fit. By then $c_{4}$ has become illdetermined and can be set to 0 . But $c_{2}$ is still very significantly different from 0 ; fixing it to 0 gives very small confidence levels (often astronomically small; in the bare coupling scheme this is even true when leaving out the $\beta=6.0$ points!).

The stability of the fit parameters also holds when leaving out large $\beta$ points. The $\Lambda$-parameter is stable on the $1 \%$ level even when leaving out all $\beta=6.2,6.4$ and 6.5 points! This clearly illustrates the quality of the fit ansatz.

We should mention that the reduced $\chi^{2}$ of the fits become very small $\left(\chi^{2} / N_{\mathrm{DF}}<0.25\right)$ once only $\beta \geq 5.85$ points are included in the fits. We take this as an indication that the errors on fine lattices have been, if anything, overestimated (at least for the data collected in table (1).

In table 6 we show results for the various fits. For definiteness we have chosen to fit $\beta=5.6-6.5$ in all cases. We now see interesting differences between the schemes: Most obviously, that the bare scheme has the largest $a^{2}$ coefficient $c_{2}$, and shows a large increase in the $\Lambda$-parameter when going from two to three loops.8 The $E$ - and $\overline{\mathrm{MS}}$ schemes, on the other hand, show much smaller $a^{2}$ corrections, and the $\Lambda$-parameter is much more stable when going from two to three loops. We regard the results in table 6

\footnotetext{
${ }^{8}$ Note that the $a^{2}$ corrections to the perturbative scaling of the string tension are not "physical", in the way that, say, $\mathrm{O}\left(a^{2}\right)$ violations of rotational symmetry would be. In fact, we could perform a (non-perturbative) change of coupling to the " $\sigma$-scheme", by defining $g_{\sigma}^{2}$ via $(a \sqrt{\sigma})(g) \equiv f\left(g_{\sigma}^{2}\right) \sqrt{\sigma} / \Lambda$. In this scheme there would be no $\mathrm{O}\left(a^{2}\right)$ corrections whatsoever.
} 
as a reconciliation of the ideas of Parisi-Lepage-Mackenzie with those of Allton.

Note that the change in going from two to three loops is significantly larger for the $V$ - than for the $E$ - or $\overline{\mathrm{MS}}$-schemes. This can be traced back to the large value of the three-loop coefficient in the scaling function, cf. table 5. This is also the reason for the jump in $c_{2}$. What is a bit surprising, perhaps, is that the results labeled $\overline{\mathrm{MS}}$, which really come from a mix of the $\overline{\mathrm{MS}}$ - and the $V$-scheme (the latter was used to extract a physical coupling from the measured plaquette), show much better agreement between the two and three loops results than the $V$-scheme. In any case, one thing does seem clear, namely, that the values of $\Lambda_{\overline{\mathrm{MS}}}$ extracted from the bare coupling scheme should not be trusted.

As another check of systematic errors we have considered using the $1 \times 2$ Wilson loop, $W_{12}$, instead of $W_{11}$, to extract $g_{P}^{2}\left(q^{\star}\right)$. Using the second order results of [33] extrapolated to infinite volume and $q^{\star}=3.0668 / a$ in a two-loop fit, we find that $\Lambda_{\overline{\mathrm{MS}}}$ goes up by $16 \mathrm{MeV}$ and $14 \mathrm{MeV}$, respectively, compared to the results of table 6 for the $V$ - and $\overline{\mathrm{MS}}$ schemes. One can also define an " $E_{12}$ "-scheme, using the expansion of $1-W_{12}$ analogously to eq. (5.1). From 33] we obtain $\Lambda_{\overline{\mathrm{MS}}} / \Lambda_{E_{12}}=27.4576$ for this scheme. Two-loop fits now show a decrease of $\Lambda_{\overline{\mathrm{MS}}}$ by $15 \mathrm{MeV}$ compared to table 6 . Unfortunately we can not perform three-loop checks for the " 12 " schemes.

Our final check of systematic errors consists of the following worst case scenario. Let us assume that for some unknown reason the true $\sqrt{\sigma}$ at both $\beta=6.4$ and 6.5 are higher or lower by $2 \%$ than the values in table 4 . First of all we find that the $\chi^{2}$ of our fits jump up by a factor of 2 or 3 (depending on the fitting interval), making the scenario appear unlikely indeed. Nevertheless, the value of $\Lambda_{\overline{\mathrm{MS}}}$ changes only by about $1-2 \%$. This was to be expected, since, as mentioned earlier, we can leave out the $\beta=6.4,6.5$ (and even 6.2) points completely from the fit, without significantly affecting the fit parameters.

As our central value for $\Lambda_{\overline{\mathrm{MS}}}$ we will quote the average of the three-loop results in the improved coupling schemes. Among these schemes, the systematic errors appear to be largest in the $V$-scheme; the difference between the two- and three-loop results, as well as switching from $W_{11}$ to $W_{12}$ to extract the coupling, give a change in $\Lambda_{\overline{\mathrm{MS}}}$ of $16 \mathrm{MeV}$. We will quote this as our error:

$$
\Lambda_{\overline{\mathrm{MS}}}=247(16) \mathrm{MeV} .
$$

This is consistent with the value recently obtained by the ALPHA collaboration [34], as well as earlier results obtained from the string tension (see e.g. [16]).

To get (5.6) we used a value of $\sqrt{\sigma}=465 \mathrm{MeV}$ for the string tension. This value is somewhat higher than the ones commonly used in the past. However, several recent studies quote a value of this size [35, 36], and we would also like to make the following simple, numerical observation. The results in table 10 show that $r_{0} \sqrt{\sigma}$ is consistent, within errors of about $1 \%$, with the string picture value

$$
r_{0} \sqrt{\sigma}=\sqrt{1.65-\frac{\pi}{12}}=1.178219 .
$$

It is generally believed [8] that $r_{0}$ is not larger than about $0.5 \mathrm{fm}$. Using $\sqrt{\sigma}=1.178 / r_{0}$ therefore gives $465 \mathrm{MeV}$ (as a lower bound) for $\sqrt{\sigma}$. 


\section{Conclusions}

We have presented a high statistics study of the static potential in pure lattice gauge theory, using the Wilson plaquette action. These data and improved analysis strategies have enabled us to give very precise determinations of the string tensions and Sommer (-type) scales for $\beta \leq 6.0$, i.e. on lattices of spacings of about $0.1 \mathrm{fm}$ and above. Combining our results with previous determinations, $a \sqrt{\sigma}$ is now known to $1 \%$ in the range $5.54 \leq \beta \leq 6.5$. According to our and other studies (quoted in sect. 4) this error also includes possible finite volume effects.

The scale $r_{0}$ is presently known somewhat less accurately on the finest lattices. Using our method to determine Sommer-type scales it should be straightforward to determine $r_{0}$ for all lattice spacings at least as accurately as the string tension. In particular, any remaining finite volume effects should be much smaller for $r_{0}$. Needless to say, our method can also be used for other kinds of gauge actions.

We have employed the ansatz of Allton 17] to provide parameterizations of $\sqrt{\sigma}$ and $r_{0}$ that are accurate to about $1 \%$ for $5.6 \leq \beta \leq 6.5$. By considering fits of this kind for the string tension in various coupling schemes we have estimated the $\Lambda$-parameter of quenched QCD to be $\Lambda_{\overline{\mathrm{MS}}}=247(16) \mathrm{MeV}$. We concluded that when it comes to extracting physical parameters, instead of just fitting data, the improved coupling schemes are very much superior to the bare coupling scheme, even when corrected for $a^{2}$ effects as suggested by Allton.

The results presented here should be useful in high precision scaling checks, comparisons of different actions, etc. Our motivation for this study has, in fact, been the desire to provide a significant check of the Symanzik improvement program by performing a scaling check of the rho mass in units of the string tension for the SW action with a nonperturbatively determined clover coefficient [5]. This is not possible without accurate knowledge of the string tension (or $r_{0}$ ). Previous estimates of the string tension would have lead to misleading conclusions about the scaling behavior of the rho mass or other observables.

\section{Acknowledgements}

This work is supported by DOE grants DE-FG05-85ER25000 and DE-FG05-96ER40979. The computations in this work were performed on the workstation cluster, the CM-2, and the new QCDSP supercomputer at SCRI. 


\section{References}

[1] C.T.H. Davies et al (eds.), LATTICE 97, Nucl. Phys. B (Proc. Suppl.), to appear.

[2] C. Bernard, M. Golterman, M. Ogilvie and J. Potvin (eds.), LATTICE 96, Nucl. Phys. B (Proc. Suppl.) 53 (1997).

[3] M. Lüscher, S. Sint, R. Sommer, P. Weisz, and U. Wolff, Nucl. Phys. B491 (1997) 323.

[4] R.G. Edwards, U.M. Heller and T.R. Klassen, hep-lat/9710054.

[5] R.G. Edwards, U.M. Heller and T.R. Klassen, in preparation.

[6] K.D. Born et al, Phys. Lett. B329 (1994) 325.

[7] K. Schilling, G.S. Bali, Int. J. Mod. Phys. C4 (1993) 1167.

[8] R. Sommer, Nucl. Phys. B411 (1994) 839.

[9] C. Michael, Phys. Lett. B283 (1992) 103.

[10] T.R. Klassen, Phys. Rev. D51 (1995) 5130.

[11] M. Lüscher, K. Symanzik and P. Weisz, Nucl. Phys. B173 (1980) 465; M. Lüscher, Nucl. Phys. B180 (1981) 317.

[12] B. Beinlich, F. Karsch, E. Laermann, and A. Peikert, hep-lat/9707023; C. Legeland et al, hep-lat/9709147.

[13] U. Glässner et al (SESAM collaboration), Phys. Lett. B383 (1996) 98.

[14] N. Cabibbo, E. Marinari, Phys. Lett. 119B (1982) 387.

[15] M. Albanese et al, Phys. Lett. 192B (1987) 163.

[16] G.S. Bali and K. Schilling, Phys. Rev. D46 (1992) 2636, Phys. Rev. D47 (1993) 661.

[17] C. Allton, hep-lat/9610016.

[18] G.S. Bali, private communication.

[19] S. Perantonis and C. Michael, Nucl. Phys. B347 (1990) 854.

[20] H. Hoeber, reanalysis of [16] with link integration; quoted in M. Göckeler et al, hep-lat/9707021.

[21] H. Wittig (UKQCD collaboration), Nucl. Phys. B (Proc. Suppl.) 42 (1995) 288; Int. J. Mod. Phys. A12 (1997) 4477.

[22] S.P. Booth et al (UKQCD collaboration), Phys. Lett. B294 (1992) 385. 
[23] M. Lüscher, R. Sommer, P. Weisz, and U. Wolff, Nucl. Phys. B413 (1994) 481.

[24] G.P. Lepage and P.B. Mackenzie, Phys. Rev. D48 (1993) 2250.

[25] G. Parisi, p. 1531 in: "High Energy Physics - 1980", L. Durand and L.G. Pondrom (eds.), AIP, New York, 1981; G. Martinelli, G. Parisi and R. Petronzio, Phys. Lett. 100B (1981) 485.

[26] L. Susskind, in "Weak and Electromagnetic Interactions at High Energy", R. Balian and C.H. Llewellyn-Smith (eds.), North Holland, Amsterdam, 1977; W. Fischler, Nucl. Phys. B129 (1977) 157.

[27] B. Alles, M. Campostrini, A. Feo and H. Panagopoulos, Phys. Lett. B324 (1994) 433; B. Alles, A. Feo and H. Panagopoulos, hep-lat/9801003.

[28] M. Lüscher and P. Weisz, Nucl. Phys. B452 (1995) 234.

[29] B. Alles, A. Feo and H. Panagopoulos, Nucl. Phys. B491 (1997) 498.

[30] M. Peter, Phys. Rev. Lett. 78 (1997) 602, Nucl. Phys. B501 (1997) 471.

[31] O.V. Tarasov, A.A. Vladimirov and A.Yu. Zharkov, Phys. Lett. 93B (1980) 429; S.A. Larin and J.A.M. Vermaseren, Phys. Lett. B303 (1993) 334.

[32] S.J. Brodsky, G.P. Lepage and P.B. Mackenzie, Phys. Rev. D28 (1983) 228.

[33] U. Heller and F. Karsch, Nucl. Phys. B251 (1985) 254.

[34] S. Capitani et al (ALPHA collaboration), hep-lat/9709125.

[35] D.W. Bliss, K. Hornbostel and G.P. Lepage, hep-lat/9605041.

[36] G.S. Bali, K. Schilling and A. Wachter, Phys. Rev. D56 (1997) 2566. 